\title{
THE STOCHASTIC LIOUVILLE EQUATION AND THE APPROACH TO THERMAL EQUILIBRIUM
}

\author{
AleXANDER J. VeGa and DANiEl Fiat
} Department of Structural Chemistry, The Weizmann Institute of Science,
Rehovot, Israel

\begin{abstract}
Some aspects of behaviour of spins in the presence of random molecular motions are discussed. Various theories which deal with the approach of the spin system to thermal equilibrium are reviewed. It is emphasized that two types of description are used. One is the ' $\rho(t)$ formalism', where the spin behaviour of one randomly moving molecule is considered. The other is the ' $\bar{\rho}(\Omega)$ formalism', where the average behaviour of all the spins which are momentarily in the same environment is described. The conventional relaxation theories make use of the $\rho(t)$ formalism, whereas the stochastic Liouville method for line shape calculations uses the $\bar{\rho}(\Omega)$ formalism. In the first type the approach to equilibrium has been dealt with for a long time. In the second type the approach to the thermal equilibrium state of $\bar{\rho}(\Omega)$ was formulated only recently in the form of the modified stochastic Liouville equation. It is pointed out that this equation has important implications for both line shape calculations and for relaxation theory.
\end{abstract}

\section{SEMI-CLASSICAL THEORY}

A. The density matrix $p(\Omega)$

This paper deals with spins which are in random motion. The simplest example of such a type of motion is that of spins which jump back and forth among a number of sites with different chemical or magnetic environment. We label the sites with the numbers $v=1,2, \ldots, n$ and define the distribution vector $p_{v}(t)$ as the vector of the probabilities of finding spins in the sites $v$ at the time $t$. Another example is the case of translational motion. The position of a spin is then characterized by the spatial coordinate $\vec{r}$ and the distribution function is $p(\vec{r}, t)$. The case of rotatory motion of molecules is very common. The orientation of the molecules is then defined by the Eulerian angles, $\Omega$. We shall treat all these cases in one formalism and choose $\Omega$ as the notation of the random coordinate. Thus the function $p(\Omega, t)$ will denote the probability density of finding spins in the environment characterized by a particular $\Omega$. We shall sometimes call this parameter 'position' or 'orientation', but this should be considered as a general term which also stands for 'site', 'conformation', etc.

To begin with, let us assume that the spins can satisfactorily be described in terms of elementary magnets $\vec{m}$, which can differ in direction but not in magnitude $|m|$. The state of the whole spin system is then given by the combined probability density $P(\Omega, m)$, which we take to be normalized: 


$$
\iint P(\Omega, \vec{m}) \mathrm{d} \Omega \mathrm{d} \vec{m}=1
$$

From this function we can extract the following quantities: the molecular distribution function:

$$
p(\Omega)=\int P(\Omega, \vec{m}) \mathrm{d} \vec{m}
$$

the distribution function of the magnetization:

$$
f(\vec{m})=\int P(\Omega, \vec{m}) \mathrm{d} \Omega
$$

the average magnetic moment:

$$
\langle\vec{m}\rangle_{0}=\iint \vec{m} P(\Omega, \vec{m}) \mathrm{d} \Omega \mathrm{d} \vec{m}
$$

the macroscopic magnetization of the sample:

$$
\vec{M}_{0}=N\langle\vec{m}\rangle_{0}
$$

where $N$ is the total number of spins per unit volume; and finally the quantities

$$
\langle\vec{m}(\Omega)\rangle=\int \vec{m} P(\Omega, \vec{m}) \mathrm{d} \vec{m}
$$

and

$$
\vec{M}(\Omega)=N\langle\vec{m}(\Omega)\rangle
$$

which may be called the $\Omega$-dependent magnetization density.

These last two quantities have the following physical meaning. Suppose that we could measure separately the total magnetization of the molecules which have their orientations between $\Omega$ and $\Omega+\mathrm{d} \Omega$. Our measurement would then yield $\vec{M}(\Omega) \mathrm{d} \Omega$. Or suppose that we could measure the total magnetization of the sample, but with an $\Omega$-dependent weight $w(\Omega)$. We would then measure $\int w(\Omega) \vec{M}(\Omega) \mathrm{d} \Omega$.

We can define $\langle\vec{m}(\Omega)\rangle$ in a slightly different way by writing

$$
P(\Omega, \vec{m})=p(\Omega) q(\Omega, \vec{m})
$$

where $q(\Omega, \vec{m})$ is the normalized probability density of finding the magnetic moment at the value $\vec{m}$, provided that we know that the molecule is in $\Omega$. We then have

$$
\langle\vec{m}(\Omega)\rangle=p(\Omega) \int \vec{m} q(\Omega, \vec{m}) \mathrm{d} \vec{m}
$$

This notation emphasizes that $\langle\vec{m}(\Omega)\rangle$ is the average magnetic moment of the molecules with orientation $\Omega$, multiplied by the probability of finding the molecules in $\Omega$. Thus $\langle\vec{m}(\Omega)\rangle$ contains information on the internal magnetic state of the molecules and on the molecular distribution in $\Omega$-space. It is important to appreciate this point when the theory is applied to systems where $p(\Omega)$ is not a uniform function, as is the case with chemical exchange between unequally populated sites, or with partially oriented molecules.

Needless to say, in the case of jumps the parameter $\Omega$ is replaced by an index $v$ and the integrations over $\Omega$ become summations.

Let us now consider molecular spin systems which are not adequately represented by a single magnetic moment $\vec{m}$. The state of the spins has then 
to be described quantum mechanically by the wave function $\psi$ or the density matrix $\rho$. As for the density matrix, we should distinguish between $\rho$ of each separate spin system (whose matrix elements $\rho_{n m}$ are the products $a_{n} a_{m}^{*}$ of the coefficients in the expansion of $\psi$ ) and its ensemble average, which we denote by $\bar{\rho}^{1,2}$. If the spin system consists of a single spin $I=\frac{1}{2}$, there is a one-to-one correspondence between $\vec{m}$ and $\rho$, and between $\langle\vec{m}\rangle$ and $\bar{\rho}^{3}$. However, if the spin system has more than two levels, this comparison cannot always be made. Nevertheless it is often convenient to visualize the behaviour of complicated spin systems through a model of magnetizations. Here we start to utilize this analogy and assume a combined probability density $P(\rho, \Omega)^{4}$, from which we derive the distribution function of the density matrices,

$$
f(\rho)=\int P(\Omega, \rho) \mathrm{d} \Omega
$$

The functions $f$ and $P$ are defined in the space of all the possible density matrices $\rho$. This space is restricted to $\rho$ s with matrix elements which are products of $a_{n}$ and $a_{m}^{*}$ taken from normalized sets of coefficients $a_{n}$. These are the matrices which satisfy the conditions of so-called pure states ${ }^{1}$. Note that the space of $\vec{m}$ was also restricted to vectors with a constant modulus $|m|$.

The determination of the functions $P(\Omega, \rho)$ and $f(\rho)$ is very difficult. However, it will turn out that we do not need an exact knowledge of their functional form, and we shall not attempt to evaluate them.

In Section II we shall adopt a complete quantum mechanical description of the whole system. This will mean that we do not need distribution functions but can work with a density matrix which is an ensemble average from the beginning.

We now come to the main subject of this introduction. We define what we. somewhat unexactly, call the $\Omega$-dependent spin density matrix

$$
\bar{\rho}(\Omega)=\int \rho P(\Omega, \rho) \mathrm{d} \rho
$$

which is the analogon of $\langle\vec{m}(\Omega)\rangle$. It may be used to compute the ensemble average of the expectation value of any physical quantity $Q(\Omega)$. which also depends on $\Omega$,

$$
\begin{aligned}
\langle Q\rangle & =\int \operatorname{tr}[\dot{\rho} Q(\Omega)] P(\Omega, \rho) \mathrm{d} \Omega \mathrm{d} \rho \\
& =\int \operatorname{tr}[\bar{\rho}(\Omega) Q(\Omega)] \mathrm{d} \Omega
\end{aligned}
$$

In most experiments performed on the spin system we measure a property $Q$ which is independent of $\Omega$. It is then sufficient to know $\bar{\rho}_{0}$ in order to calculate

$$
\langle Q\rangle=\operatorname{tr} Q \bar{\rho}_{0} ; \bar{\rho}_{0}=\int \bar{\rho}(\Omega) \mathrm{d} \rho
$$

By analogy with equation (9) we can also write

$$
\bar{\rho}(\Omega)=p(\Omega) \int \rho q(\Omega, \rho) \mathrm{d} \rho=p(\Omega) \bar{\rho}_{\Omega}
$$

which emphasizes that $\bar{\rho}(\Omega)$ is the product of the local ensemble average $\bar{\rho}_{\Omega}$ and the probability of finding molecules in $\Omega$. 


\section{B. The density matrix $\rho(t)$}

Thus far we have only considered the instantaneous description of the system of molecules and spins. However, we are actually interested in the dynamics of the system. We assume that the interactions of the spins are describable by an $\Omega$-dependent spin Hamiltonian $\mathscr{H}(\Omega)$, i.e. a Hamiltonian which is different for spins belonging to molecules with different positions. How, then, does $P(\Omega, \rho, t)$ develop in time if the molecules are moving randomly in $\Omega$-space?

The relaxation theories of Bloembergen, Purcell and Pound ${ }^{5}$, Wangness and Bloch ${ }^{6}$, Bloch $^{7,8}$ and Redfield ${ }^{9,10}$ give a partial answer to this question, in that they provide an equation of motion of the average $\bar{\rho}_{0}$ (or $\langle\vec{m}\rangle_{0}$ ). This is the so-called master equation of the density matrix ${ }^{11}$, which is valid under conditions of sufficiently rapid molecular motions.

The usual semi-classical derivation of the master question makes use of the the following concepts ${ }^{11}$. One starts to look at one particular spin system in the ensemble. Since this system belongs to a molecule which moves rapidly from one $\Omega$ to the other, a time-dependent Hamiltonian $\mathscr{H}(t)$ is observed by the spins. $\mathscr{H}(t)$ is now written as the sum of a constant $\mathscr{H}_{0}$ and a time-dependent local Hamiltonian $\mathscr{H}^{\prime}(t)$ with vanishing time average. The density matrix of the spin system in question changes according to this Hamiltonian:

$$
(\mathrm{d} / \mathrm{d} t) \rho(t)=\frac{i}{\hbar}\left[\rho(t), \mathscr{H}_{0}\right]+\frac{i}{\hbar}\left[\rho(t), \mathscr{H}^{\prime}(t)\right]
$$

This equation is first solved by following a perturbation treatment, and then the ensemble average is taken. This yields the master equation. An essential point in the averaging procedure is that the correlation between $\rho(t)$ and $\mathscr{H}(t)$ is neglected. This is only permissible if the correlation times $\tau$ of the matrix elements of $\mathscr{H}^{\prime}(t)$ are so short that

$$
\left|\mathscr{H}^{\prime}(t)\right| \tau \ll \hbar
$$

It is important to stress the difference between the density matrices $\rho(t)$ and $\bar{\rho}(\Omega) . \rho(t)$ is a pure state ${ }^{1}$ and $\bar{\rho}(\Omega)$ is an ensemble average. They yield the same average $\bar{\rho}_{0}$ upon suitable averaging, but they are not interconvertable: since the correlation with $\Omega$ is abandoned in the $\rho(t)$ formalism, $\rho(t)$ can give no information on the distribution of $\rho$ s in $\Omega$-space; and since the microscopic dynamics is not involved in $\bar{\rho}(\Omega)$, this quantity can given no information on the individual behaviour of single molecules.

A well-known difficulty related to the master equation is that its steady state solution implies an infinite spin temperature ${ }^{6-11}$. Thus the present theory does not account for the approach to thermal equilibrium. This problem can be overcome by the ad hoc assumption that $\bar{\rho}_{0}$ should be replaced by $\bar{\rho}_{0}-\bar{\rho}_{\text {eq }}$, where $\bar{\rho}_{\mathrm{eq}}$ is the Boltzmann density matrix ${ }^{11}$.

Let us now consider the analogon of equation (14) in the magnetization picture:

$$
(\mathrm{d} / \mathrm{d} t) \vec{m}(t)=\gamma \vec{m}(t) \times\left[\vec{H}_{0}+\vec{H}^{\prime}(t)\right]
$$

where $\vec{H}_{0}$ is a constant field and $\vec{H}^{\prime}(t)$ is a randomly fluctuating field. Under certain conditions (one of them being fast motion). $\left|\gamma H^{\prime}\right| \tau \ll 1$ ) it is possible 
to derive from this equation a Fokker-Planck equation for the density function $f(\vec{m}, t)^{12}$. Upon averaging, this yields rate equations for the components of the macroscopic magnetization $\vec{M}$, which can be considered as the magnetic analogon of the master equation. These equations of motion are identical with the Bloch equations, the only difference being that the term $-\vec{M}_{0} / T_{1}$ is missing, similar to the lack of $-\bar{\rho}_{\text {ea }}$ in the master equation.

The interesting aspect of equation (16) is that it can be modified to account for the term $-\vec{M}_{0} / T_{1}$ : equation (16) looks like the Langevin equation in the classical theory of Brownian motion,

$$
m \dot{u}(t)=-\beta u+F(t)
$$

where $F(t)$ is a random force and $-\beta u$ is the friction. Kubo ${ }^{12}$ proposed the addition of a friction force to equation (16), and wrote

$$
(\mathrm{d} / \mathrm{d} t) \vec{m}(t)=\gamma \vec{m}(t) \times\left(\vec{H}_{0}+\vec{H}^{\prime}(t)\right)-\eta \vec{m} \times\left(\vec{m} \times \vec{H}_{0}\right)
$$

where the friction force is of the Landau-Lifschitz type ${ }^{13}$. A friction of the form $-\beta \vec{m}$ might seem more obvious ${ }^{14}$, but cannot be correct, because $|m|$ should remain constant. The value of the friction coefficient $\eta$ is assumed to be related to the random field in a way similar to the Einstein relation between $\beta$ and $F(t)$. In the limit of extremely fast motion $\left(\gamma\left|H_{0}\right|, \gamma\left|H^{\prime}\right| \ll 1 / \tau\right)$ this leads to the complete Bloch equations.

This treatment is perhaps the most elementary justification of the ad hoc corrections of the master equation. It has a purely phenomenological character, since no microscopic justification is given for the friction force. But even when the friction term is taken for granted, it is impossible to deal with a finite correlation time $\tau^{12}$. The development of a Brownian motion theory on the basis of equation (18) is very difficult, owing to the non-linearity of this equation ${ }^{15}$. The problem becomes even more complicated if one tries to solve the quantum mechanical analogon for $\rho(t)$.

\section{The stochastic Liouville equation}

Equation (14) is a stochastic equation which defines the stochastic process $\rho(t)$ in terms of the stochastic process $\mathscr{H}(t)$. More precisely, by writing

$$
(\mathrm{d} / \mathrm{d} t) \rho(t)=\frac{i}{\hbar}\left[\rho(t), \mathscr{H}_{0}+\mathscr{H}^{\prime}\{\Omega(t)\}\right]
$$

we see that the process $\rho(t)$ actually depends on the stochastic process $\Omega(t)$. In most problems dealt with in magnetic resonance theory some model is assumed for the description of the stochastic process $\Omega(t)$. In nearly all cases this is a stationary Markoffian process. It is then assumed that the probability density $p(\Omega, t)$ satisfies the equation

$$
(\partial / \partial t) p(\Omega, t)=\Gamma p(\Omega, t)
$$

where $\Gamma$ is a time-independent Markoffian operator, operating on functions of $\Omega$. More generally, $\Omega(t)$ is the projection of a Markoffian process, i.e. $\Omega$ should be supplemented with additional variables to form a complete set of random variables which make a Markoffian process. In order to retain 
a simple notation we assume that $\Omega$ itself is a Markoffian process. Equation (20) fits well in the formalism of Section A but it can be less directly applied to further development of equation (14). Thus we follow Kubo's development ${ }^{4}$ and write formally for the rate equation of $P(\Omega, \rho, t)$

$$
\frac{\partial}{\partial t} P(\Omega, \rho, t)=\left\{-\frac{\partial}{\partial \rho} \frac{i}{\hbar}[\rho, \mathscr{H}(\Omega)]+\Gamma\right\} P(\Omega, \rho, t)
$$

This can be regarded as a composite Markoffian process. It is a coarsegrained description of the complete Liouville equation of the density of states of the combined system of lattice and spins, utilizing the stochastic property $\Gamma$. Thus we may call it a stochastic Liouville equation ${ }^{4,16}$.

We first multiply equation (21) by $\rho$ and integrate over $\rho$. This yields, with equation (11),

$$
(\partial / \partial t) \bar{\rho}(\Omega, t)=\frac{i}{\hbar}[\bar{\rho}(\Omega, t), \mathscr{H}(\Omega)]+\Gamma \bar{\rho}(\Omega, t)
$$

This is the equation which we shall refer to as the stochastic Liouville equation (SLE).

In this derivation of the SLE an important approximation has been made. It is assumed that the molecules execute their random motions regardless of the state in which the spins find themselves. Thus the reaction of the spin system to its surroundings is ignored. In other words. we neglected the energy exchange between the lattice and the spins. However, as Kubo stated $^{4}$, 'this is permissible, for instance, when the temperature of the bath is sufficiently high compared with the possible energy exchange. Many examples in NMR or Mössbauer effects belong to this category because the reaction to the molecular motion of the bath is extremely small.' Thus equation (22) has a wide range of application in line shape problems in magnetic resonance ${ }^{16}$.

In the case of jumps the operator $\Gamma$ is a matrix and the SLE takes the form

$$
(\mathrm{d} / \mathrm{d} t) \bar{\rho}_{v}=\frac{i}{\hbar}\left[\bar{\rho}_{v}, \mathscr{H}_{v}\right]+\sum_{\mu} \Gamma_{v \mu} \rho_{\mu}
$$

where $\Gamma_{\nu \mu}$ are reciprocals of mean residence times.

The SLE for the magnetization is derived in the same way, and the analogons of equations $(22,23)$ are ${ }^{16,17}$

$$
(\partial / \partial t) \vec{M}(\Omega, t)=\gamma \vec{M}(\Omega, t) \times \vec{H}(\Omega)+\Gamma \vec{M}(\Omega, t)
$$

and

$$
(\mathrm{d} / \mathrm{d} t) \vec{M}_{v}(t)=\gamma \vec{M}_{v}(t) \times \vec{H}_{v}+\sum_{\mu} \Gamma_{v \mu} \vec{M}_{\mu}(t)
$$

Obviously, equations (22)-(25) can suitably be extended with phenomenological relaxation terms. However, one must realize that the relaxation processes introduced through such terms must originate from stochastic processes other than the one represented by the operator $\Gamma$.

Equations (23) and (25) have been derived earlier in several ways and are extensively used in the theory of the effect of chemical rate processes on 
magnetic resonance. The theory and the applications of these equations are reviewed in an article by Johnson ${ }^{18}$.

The situation where $\Omega$ is a continuous variable has found application only in recent years, with one exception: In 1956 Torrey ${ }^{19}$ derived rate equations, similar to equation (24), for spins undergoing translatory diffusion. These are the Bloch equations with diffusion terms, which found important application in the spin echo technique.

Like the master equation, the stochastic Liouville equation has the shortcoming that its stationary solution implies an infinite temperature. This is closely related to the neglect of the reaction of the spins to the random motions. It has been suggested ${ }^{16}$ that the main reason for the difficulty of this problem lies in our inability to solve a non-linear quantum mechanical Langevin equation, as we mentioned at the end of the previous section. However, we feel that even if such a Langevin equation could be dealt with, this would not help us to properly correct the stochastic Liouville equation, since the Langevin equation belongs to the $\rho(t)$ formalism, whereas the stochastic Liouville equation is a rate equation of $\bar{\rho}(\Omega)$. In fact, in Section II we shall point out that an appropriate rectification of equation (22) in terms of a $\bar{\rho}(\Omega)$ description can be made.

\section{Torrey's diffusion equations}

In the case of translational diffusion in an inhomogeneous magnetic field $\vec{H}(\vec{r})$, the random molecular coordinate is the displacement vector $\vec{r}$ and the operator $\Gamma$ becomes $\nabla \cdot D \nabla$, where $D$ is the diffusion coefficient. Thus equation (24) becomes

$$
(\partial / \partial t) \vec{M}(\vec{r}, t)=\gamma \vec{M}(\vec{r}, t) \times \vec{H}(\vec{r})+\nabla \cdot D \nabla \vec{M}(\vec{r}, t)
$$

As mentioned above, the rate equation of $\vec{M}(\vec{r})$ was first given by Torrey ${ }^{19}$. His derivation was based on the theory of Brownian motion and the result was

$$
\begin{aligned}
& \frac{\partial}{\partial t} M_{x}(\vec{r}, t)=\gamma(\vec{M} \times \vec{H})_{x}-\frac{M_{x}}{T_{2}}+\nabla \cdot D \nabla\left(M_{x}-M_{x \mathrm{eq}}\right) \\
& \frac{\partial}{\partial t} M_{y}(\vec{r}, t)=\gamma(\vec{M} \times \vec{H})_{y}-\frac{M_{y}}{T_{2}}+\nabla \cdot D \nabla\left(M_{y}-M_{y \mathrm{eq}}\right) \\
& \frac{\partial}{\partial t} M_{z}(\vec{r}, t)=\gamma(\vec{M} \times \vec{H})_{z}-\frac{M_{z}-M_{z \mathrm{eq}}}{T_{1}}+\nabla \cdot D \nabla\left(M_{z}-\mathrm{M}_{z \mathrm{eq}}\right)
\end{aligned}
$$

These equations are the same as equation (26), but supplemented with extra relaxation terms and an inhomogeneous term $-\nabla . D \nabla \vec{M}_{\mathrm{eq}}(\vec{r})$. Here $\vec{M}_{\mathrm{eq}}(\vec{r})$ is the $\vec{r}$-dependent equilibrium magnetization,

$$
\vec{M}_{\text {eq }}(\vec{r})=\chi_{0} \vec{H}(\vec{r})
$$

$\chi_{0}$ being the static susceptibility. The origin of the various terms in Torrey's equations is as follows. The first term describes the change of the magnetization within each molecule due to the Larmor precession about the local field $\vec{H}(\vec{r})$. The term $\nabla \cdot D \nabla \vec{M}$ represents the change in the spatial 
distribution of the magnetization due to the Brownian motion of the molecules. The inhomogeneous term $-\nabla \cdot D \nabla \vec{M}_{\text {eq }}$ results from the force which acts upon the magnetic moments of the molecules which move in an inhomogeneous magnetic field: since molecules which have their spins in opposite directions are drawn to different regions of the magnetic field, a net 'drift current' of the magnetization results.

In the absence of the relaxation terms the 'drift terms' ensure that the magnetization approaches its equilibrium value. Thus Torrey's equations can serve as a simple classical explanation for the approach to a finite spin temperature. Interestingly, Torrey's article ${ }^{19}$ appeared almost simultaneously with the quantum mechanical relaxation theories of Bloch ${ }^{7}$ and Redfield 9 . These rather complicated theories give a fundamental justification of the ad hoc modification of the master equation by which the approach to equilibrium is established. Apparently these authors thought that the use of a quantum mechanical description of the lattice was the only practical way of justifying the ad hoc assumption.

It is surprising that Torrey's 'drift terms' were never considered an important aid to the understanding of the process of relaxation towards equilibrium. We can think of two reasons why this explanation remained unnoticed. One is that, in the case where $M_{\mathrm{eq}} / T_{1} \geqslant \nabla . D \nabla M_{\mathrm{eq}}$, the 'drift terms' have no effect on the solutions of equation (27). Since this is the case in the situations where the equations are applied (spin echo experiments) ${ }^{19}$, these terms were generally overlooked. Secondly, the lack of communication between the two theories might stem from 'language difficulties': the conventional relaxation theory used the $\rho(t)$ formalism, whereas equation (27) is written in the $\bar{\rho}(\Omega)$ formalism (see above).

\section{QUANTUM MECHANICAL THEORY}

\section{A. $\rho(\Omega)$ in equilibrium}

In a previous publication ${ }^{20}$ we used a formalism where quantities such as $p(\Omega), \Gamma, \rho(\Omega)$ were defined quantum mechanically. This formalism permitted calculation of $\rho(\Omega)$ in thermal equilibrium in the high-temperature approximation. The first-order result was

$$
\rho_{\text {eq }}(\Omega)=p_{\text {eq }}(\Omega) A^{-1}[1-\mathscr{H}(\Omega) / k T]
$$

where $p_{\text {eq }}$ is the equilibrium distribution and $A$ is the number of spin states. Thus $\rho_{\mathrm{eq}}(\Omega)$ is, at least to first order, equal to the $\Omega$-dependent Boltzmann density matrix

$$
p_{\mathrm{B}}(\Omega)=p_{\text {eq }}(\Omega) \exp [-\mathscr{H}(\Omega) / k T] / \operatorname{tr} \exp [-\mathscr{H}(\Omega) / k T]
$$

Furthermore, the stochastic Liouville equation could be derived quantum mechanically. This derivation was to a large extent inspired by the quantum mechanical derivation of the master equation, and, indeed, led to a modification of the SLE which accounts for the approach to thermal equilibrium ${ }^{20}$ :

$$
(\partial / \partial t) \rho(\Omega, t)=i \hbar^{-1}[\rho(\Omega, t), \mathscr{H}(\Omega)]+\Gamma\left\{\rho(\Omega, t)-\rho_{\mathrm{eq}}(\Omega)\right\}
$$

Since $\rho_{\text {eq }}$ commutes with $\mathscr{H}(\Omega)$, it is easily seen that $\rho(\Omega)=\rho_{\text {eq }}(\Omega)$ is the 
steady state solution of this equation. Clearly Torrey's equations are a special case of this equation. For the details of the derivations we refer to the original paper ${ }^{20}$. Here we confine ourselves to some general remarks. For the sake of comparison we recall the assumptions and main features of the derivation of the master equation.

\section{B. Master equation}

There exist three derivations of the master equation with slight differences between them. i.e. those of Wangness and Bloch 6 . Redfield ${ }^{9} \cdot{ }^{10}$ and Abragam $^{21}$. We take the latter as the most suitable example for our treatment.

The total Hamiltonian is

$$
\mathscr{H}_{\mathrm{T}}=\mathscr{H}_{\mathrm{L}}+\mathscr{H}_{\mathrm{S}}=\mathscr{H}_{\mathrm{L}}+\mathscr{H}_{0}+\mathscr{H}_{1}
$$

where $\mathscr{H}_{0}$ is a pure spin Hamiltonian and $\mathscr{H}_{1}$ is the perturbing coupling between the lattice and the spins. $\mathscr{H}_{1}$ can be expanded as

$$
\mathscr{H}_{1}=\sum_{q} F_{q} A_{q}
$$

where the $F_{q}$ and the $A_{q}$ are lattice and spin operators, respectively. The total density matrix of the combined system of lattice and spins, $\sigma_{\mathrm{T}}$, is assumed to be of the form

$$
\sigma_{\mathrm{T}}=\sigma_{\mathrm{L}} \rho
$$

where $\sigma_{\mathrm{L}}$ is the lattice density matrix and $\rho$ is the spin density matrix

$$
\left\langle m|\rho| m^{\prime}\right\rangle=\sum_{i}\left\langle i m\left|\sigma_{\mathrm{T}}\right| i m^{\prime}\right\rangle
$$

where $|i\rangle$ denotes a lattice state and $|m\rangle$ a spin state. The fundamental assumption is that the lattice, because of its very large heat capacity, remains in thermal equilibrium, so that $\rho_{\mathrm{L}}=\rho_{\text {Leq }}$.

The equation of motion for $\rho_{\mathrm{T}}$ is transformed to the interaction representation,

$$
(\mathrm{d} / \mathrm{d} t) \sigma_{\mathrm{T}}^{*}=\frac{i}{\hbar}\left[\sigma_{\mathrm{T}}^{*}, \mathscr{H}_{1}^{*}(t)\right]
$$

This equation is integrated by successive approximations up to the second order. The trace over $i$ gives

$$
(\mathrm{d} / \mathrm{d} t) \rho^{*}=-\operatorname{tr}_{i} \int_{0}^{t}\left[\mathscr{H}_{1}^{*}(t),\left[\mathscr{H}_{1}^{*}(t-\tau), \sigma_{\mathrm{T}}^{*}(0)\right]\right] \mathrm{d} t
$$

Now the following assumptions are successively made. The correlation times $\tau_{\mathrm{c}}$ of the matrix elements of $F_{q}^{*}(t)$ are so short that

$$
\tau_{\mathrm{c}}\left|\mathscr{H}_{1}\right| \ll \hbar
$$

Further,

$$
\left|\mathscr{H}_{0}\right| \ll k T
$$

and

$$
\left|\rho-\frac{1}{A}\right| \ll 1
$$


This leads to the master equation,

$$
\mathrm{d} \rho^{*} / \mathrm{d} t=-\frac{1}{2} \operatorname{tr}_{i} \int_{-\infty}^{\infty}\left[\mathscr{H}_{1}^{*}(t),\left[\mathscr{H}_{1}^{*}\left(t^{\prime}\right), \rho^{*}-\rho_{\text {eq }}\right]\right] \sigma_{\text {Leq }} \mathrm{d} t^{\prime}
$$

where the relaxation is clearly towards the Boltzmann density matrix

$$
\rho_{\text {eq }}=A^{-1}\left[1-\mathscr{H}_{0} / k T\right]
$$

The equation is further transformed to a more useful expression, containing Fourier transforms of the correlation functions

$g_{q q^{\prime}}(t)=\frac{1}{Z} \sum_{i f}\left\langle i\left|F_{q}\right| f\right\rangle\left\langle f\left|F_{-q}^{\prime}\right| i\right\rangle \exp \left[i\left(E_{f}-E_{i}\right) t / \hbar\right] \exp \left(-E_{i} / k T\right)$

The main problem in statistical mechanics of irreversible processes is to obtain the rate equation (or transport coefficient) of an irreversible process from the deterministic classical Hamilton equations or the quantum mechanical Schrödinger equation ${ }^{22}$. In our particular case, the problem is to deduce the irreversible relaxation equations from the deterministic equation (36). The present derivation does not give a fundamental solution of this problem. Instead it is merely assumed that the lattice behaves in an irreversible way. The irreversibility of the lattice is then utilized in equation (43). In this equation we identify the deterministic expression at the righthand side with the phenomenological correlation function $g_{q q^{\prime}}(t)$. Through this replacement irreversibility is introduced. since $g_{q q^{\prime}}(t)$ is assumed to tend to zero for $t \gg \tau_{\mathrm{c}}$. (The same assumption was also made in the application of the condition of equation 38.) In order that we may use equation (43), it is further assumed that the lattice remains in equilibrium, such that the way in which it manifests itself in $g_{q q^{\prime}}(t)$ remains unaltered throughout the entire process.

Finally, we notice that in most cases the practical evaluation of the correlation times is taken from some coarse-grained classical description of the lattice motions; mostly a Markoffian process ${ }^{10,23,24}$.

\section{The stochastic Liouville equation}

The assumptions made in the quantum mechanical derivations of the $\mathrm{SLE}^{20}$ are summarized in Table 1, alongside the corresponding assumptions of the master equation.

Both theories assume a high temperature (assumption 1) and separability of the total $\sigma_{\mathrm{T}}$ into the equilibrium lattice density matrix $\sigma_{\mathrm{Leq}}$ and a spin density matrix $\sigma_{\mathrm{S}}$ or $\rho$ (assumption 2). They differ in that $\rho$ is a pure spin operator (equation 35), whereas $\sigma_{\mathrm{S}}$ operates in the space of the lattice as well as in that of the spins. This difference is the reason why the SLE can deal with the correlation between the state of the lattice and that of the spins, whereas the master equation cannot: in the formalism of $\rho(\Omega)$ we have different spin states for different $\Omega \mathrm{s}$, whereas in the formalism of the master equation no such differentiation can be made.

Weak coupling between lattice and spins is expressed by Van Vleck's condition $^{20}$. This assumption states that only pairs of lattice states whose energy differences are smaller than $k T$ are mixed by the spin Hamiltonian. 
Table 1. The assumptions made in the quantum mechanical derivations of the stochastic Liouville equation and the master equation

\begin{tabular}{|c|c|}
\hline SLE & Master equation \\
\hline 1. $|\mathscr{H}(\Omega)| \ll k T$ & $\left|\mathscr{H}_{0}\right| \ll k T$ \\
\hline 2. $\sigma_{\mathrm{T}}=\sigma_{\mathrm{Leq}} \sigma_{\mathrm{S}}$ & \\
\hline 3. Van Vleck's condition & $\sigma_{\mathrm{T}}=\sigma_{\mathrm{Leq}} \rho$ \\
\hline 4. $\quad \sigma_{\mathrm{s}}=\sum_{q} R_{q}(\Omega) B_{q}$ & \\
\hline 5. $\left|\sigma_{S}-1 / A\right| \ll 1$ & $|\rho-1 / A| \ll 1$ \\
\hline 6. $\quad(\partial / \partial t) p(\Omega, t)=\Gamma p(\Omega, t)$ & $g_{q q^{\prime}}(\tau)=\left\langle F_{q^{\prime}}(t) F_{-q^{\prime}}(t+\tau)\right\rangle$, equation $(43)$ \\
\hline 7. $\mathscr{H}(\Omega)=\sum_{q} F_{q}(\Omega) A_{q}$ & - \\
\hline 8. - - & $\tau_{\mathrm{c}}\left|\mathscr{H}_{1}\right| \ll \hbar$ \\
\hline
\end{tabular}

Van Vleck's condition is not explicitly formulated in the master equation theory, but it is implied in the assumption $\sigma_{\mathrm{S}}=\rho^{25}$.

Assumption 4 means that the spin part of the density matrix can be written as the sum of products of algebraic functions of $\Omega$ and pure spin operators. Obviously, also, assumption 4 is implied in $\sigma_{\mathrm{S}}=\rho$.

The two theories agree in that they assume a small deviation of the spin density matrix from uniformity (assumption 5). The final similarity is found in the assumption of a certain coarse-grained description of the lattice motions (assumption 6). Also, in the SLE derivation this is the point where irreversibility is introduced through the replacement of a deterministic expression by a phenomenological stochastic expression, involving the Markoffian operator $\Gamma^{20}$. As we have already remarked in connection with equation (43), in most applications of the master equation the correlation functions are calculated from some assumed Markoffian process. Assumptions 5 are then identical.

The last two lines of Table 1 indicate the difference between the applicabilities of the two rate equations. The SLE is only valid if the spin Hamiltonian consists of $\Omega$-dependent spin operators (assumption 7 ), but, as we have remarked, in nearly all relaxation mechanisms of practical significance this is the case. The only important difference then remains the requirement of short correlation times for the validity of the master equation assumption. Therefore the master equation should follow from the SLE if we supplement it with the condition of short correlation times. In another publication it will be shown that this, indeed, is the case.

\section{CONCLUSION}

Until recently two problems in the theory of magnetic resonance were unsolved: the question of the orientational dependence of the equilibrium spin density matrix $\rho_{\text {eq }}(\Omega)$ in tumbling molecules; and the problem of the inability of the stochastic Liouville equation (SLE) to describe the approach to $\rho_{\mathrm{eq}}(\Omega)$. A solution to both problems is now found in the form of the modified SLE. 
The SLE in its modified form is more versatile than the original equation, because it is able to deal with situations where the spin system is far removed from equilibrium. This has consequences for the theory of line shape calculations ${ }^{27}$ as well as for the relaxation theory ${ }^{26}$.

The SLE and the $\bar{\rho}(\Omega)$ formalism are usually applied to the calculation of line shapes, especially in the slow motion limit ${ }^{16,17}$. It can be shown that line shape calculations find a more reliable basis in the modified equation and that saturation effects can now easily be treated ${ }^{27}$.

Since the modified SLE accounts for the approach to equilibrium, it is a suitable tool for the description of relaxation mechanisms. This new approach $^{26}$ to the theory of relaxation shows that the $\bar{\rho}(\Omega)$ formalism is also of value for this branch of magnetic resonance theory. We even believe that the $\bar{\rho}(\Omega)$ derivation of the relaxation formulas has several conceptual advantages over the conventional $\rho(t)$ derivations. These have to do with the correlation between the states of the spin system and the surroundings, which is retained in the $\bar{\rho}(\Omega)$ description but abandoned in the $\rho(t)$ picture.

\section{REFERENCES}

${ }^{1}$ R. G. Tolman, The Principles of Statistical Mechanics, p 327 ff. Oxford University Press: New York (1938).

2 C. P. Slichter, Principles of Magnetic Resonance, p 127. Harper and Row: New York (1963).

3 A. Abragam, The Principles of Nuclear Magnetism, p 36. Clarendon Press: Oxford (1961),

${ }^{4}$ R. Kubo, J. Phys. Soc. Japan, 26, Suppl., 1 (1969).

5 N. Bloembergen, E. M. Purcell and R. V. Pound, Phys. Rev. 73, 679 (1948).

6 R. K. Wangness and F. Bloch, Phys. Rev. 89, 728 (1953).

7 F. Bloch, Phys. Rev. 102, 104 (1956).

${ }^{8}$ F. Bloch, Phys. Rev. 105, 1206 (1957).

9 A. G. Redfield, I.B.M. Journal, 1, 19 (1957).

10 A. G. Redfield, Adv. Mag. Res. 1, 1 (1965).

11 Ref. 3, p 276 ff.

12 R. Kubo, Int. Symposium on NMR, Preliminary Report, N-1-2. Tokyo (1965).

13 L. D. Landau and E. M. Lifschitz, The Classical Theory of Fields, §75. Pergamon: Oxford (1962).

14 J. M. Deutsch and I. Oppenheim, Adv. Mag. Res. 3, 43 (1968).

15 N. G. van Kampen, Adv. Chem. Phys. 15, 65 (1969).

16 R. Kubo, Adv. Chem. Phys. 15, 101 (1969).

${ }^{17}$ R. Kubo and T. Toyabe, Magnetic Resonance and Relaxation (Proc. XIV-ième Colloque Ampère), p 810. North-Holland: Amsterdam (1967).

18 C. S. Johnson Jr, Adv. Mag. Res. 1, 33 (1965).

19 H. C. Torrey, Phys. Rev. 104, 563 (1956); Ref. 3, p 60.

20 A. J. Vega and D. Fiat, J. Chem. Phys. 60, 579 (1974).

21 Ref. 3, p 283.

22 I. Prigogine. Non-Equilibrium Statistical Mechanics. Interscience: New York (1962).

23 Ref. 3, p 298.

24 Ref. 2, p 230.

25 Ref. 3, p 288, the equation following equation (66" ').

26 A. J. Vega and D. Fiat, Relaxation Theory and the Stochastic Liouville Equation. To be published.

27 A. J. Vega and D. Fiat, J. Mag. Res. 13, 260 (1974). 